\title{
Recent Development to Explore the Use of Biodegradable Periodic Mesoporous Organosilica (BPMO) Nanomaterials for Cancer Therapy
}

\author{
Shanmugavel Chinnathambi ${ }^{1}$ (i) and Fuyuhiko Tamanoi ${ }^{1,2, *(1)}$ \\ 1 Institute for Integrated Cell-Material Sciences, Institute for Advanced Study, Kyoto University, \\ Kyoto 606-8501, Japan; chinnathambi.shanmugavel.8s@kyoto-u.ac.jp \\ 2 Department of Microbio., Immunol. \& Molec. Genet., University of California, Los Angeles, CA 90095, USA \\ * Correspondence: tamanoi.fuyuhiko.2c@kyoto-u.ac.jp; Tel.: +81-75-753-9856
}

Received: 26 August 2020; Accepted: 15 September 2020; Published: 18 September 2020

check for updates

\begin{abstract}
Porous nanomaterials can be used to load various anti-cancer drugs efficiently and deliver them to a particular location in the body with minimal toxicity. Biodegradable periodic mesoporous organosilica nanoparticles (BPMOs) have recently emerged as promising candidates for disease targeting and drug delivery. They have a large functional surface and well-defined pores with a biodegradable organic group framework. Multiple biodegradation methods have been explored, such as the use of redox, $\mathrm{pH}$, enzymatic activity, and light. Various drug delivery systems using BPMO have been developed. This review describes recent advances in the biomedical application of BPMOs.
\end{abstract}

Keywords: PMOs; biodegradable; drug delivery; cancer targeting and treatment

\section{Introduction}

Cancer is one of the most life-threatening diseases; it affects millions of people every year [1]. While the number of cases has decreased due to excellent diagnosis and treatment methods, the problem is that the current treatments kill healthy cells during chemotherapy, surgery, and radiation therapy. Targeting therapy is one of the best methods to reduce side effects. So, nanomaterials provide ideal ways to carry drugs to the targeted area without affecting healthy tissues. In the context of solid tumors that have a rich vasculature, the enhanced permeability and retention (EPR) effect can be exploited for cancer targeting $[2,3]$. In recent years, various nanomaterials were developed for biomedical solutions such as diagnosis and targeted drug delivery [4-10].

Mesoporous silica nanoparticles (MSNs) have emerged as a promising type of nanoparticles. They consist of an Si-O-Si framework with multiple pores generated by using surfactants such as cetyltrimethylammonium bromide (CTAB) as a templating agent. Because of the presence of the pore surface, these materials possess a large surface area where a variety of chemicals can be attached. In addition, their relative stability enables various chemical modifications to be carried out. Thus, sol-gel derived inorganic porous materials have become promising new carriers in drug delivery systems that have a high surface area, are well defined and tunable, and possess a uniform pore structure and biocompatibility [11-16].

Of particular interest are biodegradable periodic mesoporous organosilica nanoparticles (BPMOs) that were developed to enhance nanoparticle degradation in biological systems by incorporating a biodegradable organic bridge between two silicon atoms. BPMOs are a type of PMO, a unique class of materials that possess periodically ordered pores and nanometer-thick pore walls made of organosilica, in which each organic group is covalently bonded to two or more silicon atoms [17-23]. Because 
of their advanced structural features, PMOs are used for a broad range of applications [9,24-28]. In biomedical applications, fluorescently modified PMOs play critical roles in cell imaging and tumor targeting $[25,29,30]$. Several review papers have been published about the synthesis and applications of PMOs [16-19,21,22,24,25,27]. In this review, we focused on recent work that used BPMO systems for biodegradation studies, drug delivery, and anti-cancer treatment.

\section{PMO and BPMO}

MSNs use tetraalkoxysilane (TEOS) as a precursor to form the Si-O-Si framework. On the other hand, PMOs [13,31,32] are prepared by using bridged alkoxysilane (Figure 1). In PMO, bi-silane has a functional organic bridge $(\mathrm{R})$ between silicon atoms, like $\left(\mathrm{R}^{\prime} \mathrm{O}\right) 3 \mathrm{Si}-\mathrm{R}-\mathrm{Si}\left(\mathrm{R}^{\prime} \mathrm{O}\right) 3$, and $\mathrm{OR}$, representing a methoxy or ethoxy group (Figure 1). Those structures create remarkable advantages, such a as flexible framework, homogeneous distribution of organic units, and the convenient entrance of pore channels. Vercaemst et al. synthesized diastereoisomerically pure E-isomers and Z-isomers of 1,2-bis(triethoxysilyl)ethane for the development of PMO [33]. Asefa et al. synthesized PMO with an organic group inside the channel wall; PMOs contain bridge-bonded ethene groups directly integrated into the silica framework. This approach created a stable and periodic mesoporous ethene silica with high surface area and ethene groups that are readily accessible for chemical reactions [34]. Hoffmann et al. reviewed PMOs' synthetic chemistry and its applications [22].

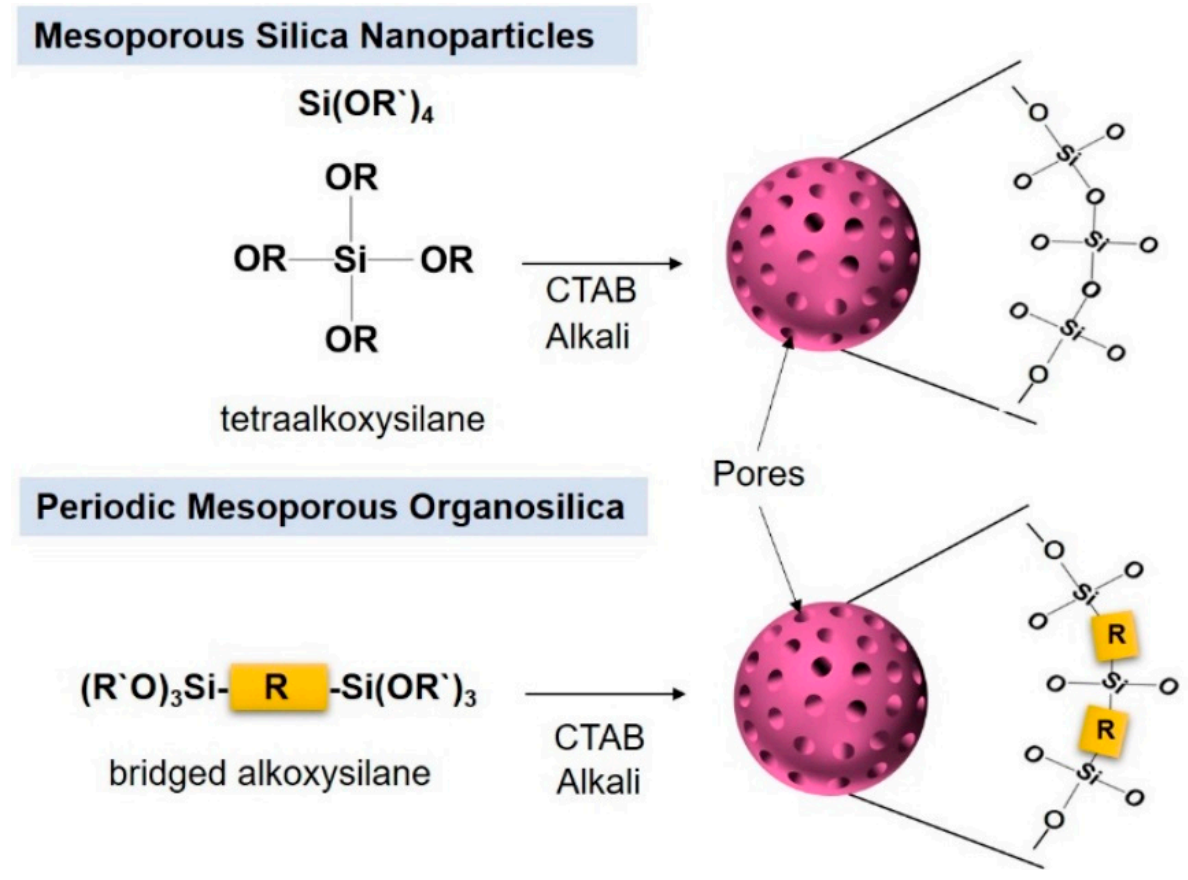

Figure 1. Schematic representation of mesoporous silica nanoparticles (MSNs) and periodic mesoporous organosilica nanoparticles (PMOs) with precursors.

In the case of BPMOs, $\mathrm{R}$ in the precursor is used to design biodegradability into PMO. This is prepared by using a bridged alkoxysilane precursor that contains biodegradable chemical bonds such as ones that respond to redox conditions, low-pH conditions, enzyme conditions, etc. It is necessary to select an ideal degradable linker between two silicon atoms depending on the application of the nanomaterial. BPMOs are receiving significant attention in the development of nanomedical research and applications [10]. Biodegradation can be defined as the degradation caused by various conditions resulting from cell activities $[16,35,36]$. According to the US Food and Drug Administration (FDA), it appears that all injected agents need to be cleared from the body in a reasonable time period. A globular nanoparticle (NP) with a hydrodynamic diameter (HD) less than $6 \mathrm{~nm}$ can easily pass through the glomerular capillary wall, while it is difficult for those with a diameter more than $8 \mathrm{~nm}$ to 
cross through [37]. BPMOs can be designed to depend on internal conditions such as redox potential, $\mathrm{pH}$ conditions, presence of enzymes, temperature change, as well as on external stimuli such as light and ultrasound exposure.

It should be noted that $\mathrm{R}$ can also be used to enhance the loading and release of anti-cancer drugs. The loading of anti-cancer drugs can be significantly increased by various interactions created between the cargo and the nanoparticle. In addition, the release of anti-cancer drugs can be enhanced by a change in conditions, including low $\mathrm{pH}$, redox conditions, and light exposure. By exploiting this feature, a variety of systems that respond to internal or external conditions have been developed.

\section{Redox Responsive Systems}

Redox cleavage of the sulfide bridges by an intracellular reducing agent such as glutathione (GSH) has been used for biodegradation. The idea to use redox conditions for biodegradation is based on the observation that the intracellular GSH level is much higher than that in the bloodstream (the intracellular GSH concentration can reach $10 \mathrm{mM}$, while the extracellular concentration is $10 \mu \mathrm{M}$ ). Thus, this type of $\mathrm{BPMO}$ is expected to remain intact during circulation in the bloodstream but will be degraded upon cellular uptake. Croissant et al. [13] reported biodegradable ethylene-bis(propyl) disulfide (EDIS)-based PMO nanorods and nanospheres for efficient in vitro drug delivery. Mixed PMO is controlled by the co-condensation ratio between bis(triethoxysilyl)ethylene and bis(3-triethoxysilylpropyl) disulfide. Michigan Cancer Foundation-7 (MCF-7) cell lines show perfect compatibility and biodegradability with physiological conditions when using EDIS NPs. The loading capacity of doxorubicin (DOX) in acidic conditions appears high in comparison with neutral conditions, and DOX is released in the lysosome upon stimulation with acidic stimuli. Additionally, the nanoparticles are localized after $24 \mathrm{~h}$ incubation. According to the above results, both materials acted as strong biodegradable Trojan horses for in vitro cancer therapy.

Vu et al. prepared BPMOs with tetrasulfide bonds and demonstrated their excellent capability to deliver doxorubicin. In this experiment, we used BPMO NPs carriers to deliver the anti-cancer drugs to a chicken chorioallantoic membrane (CAM) assay. Here, human ovarian carcinoma (OVCAR-8) cells on the CAM membrane of the fertilized egg were used to form the tumor. Later, DOX-loaded BPMO NPs were injected intravenously into the chicken egg to eliminate the tumor. No damage to the various organs present in the chicken embryo was observed. In opposition, widespread organ damage was observed when free DOX was used. The toxicity of the drug is dependent on the nanoparticle-associated delivery [38].

Recently, Mai et al. prepared BPMO nanoparticles containing tetrasulfide bonds by using two bridged alkoxysilane precursors, one of which included bridged tetrasulfide units. The BPMOs could be degraded completely after incubation with $10 \mathrm{mM}$ GSH for three days, as demonstrated by microscopic observation as well as by dynamic light scattering (DLS) measurements (Figure 2) in both phosphate-buffered saline (PBS) and in simulated body fluid (SBF). Here, the authors used two models (tumor spheroids and the chicken egg tumor model) to evaluate the daunorubicin (DNR) as an anti-cancer drug. A very good accumulation of BPMO in the chicken egg model was observed, and this increased the uptake of the drug with a less toxic side effect. DNR BPMOs will be very good clinical candidates in the future [39].

Moghaddam et al. synthesized and characterized highly uniform redox-responsive polysulfide-based biodegradable silica nanoparticles that differ in size (58 to $332 \mathrm{~nm}$ ), porosity, and composition (Figure 3a). These particles include mesoporous $\mathrm{D}$ that contains disulfide bonds, mesoporous $\mathrm{T}$ that contains tetrasulfide bonds as well as nonporous $\mathrm{D}$ that contains disulfide bonds. The release of DOX was analyzed in the presence and absence of GSH (Figure 3b). The degradation study demonstrated that the mesoporous nanoparticles underwent surface and bulk erosion and were degraded after incubation with $8 \mathrm{mM}$ of GSH. Importantly, mesoporous nanoparticles had a higher degradation rate than nonporous nanoparticles, suggesting that the higher surface area of the mesoporous nanoparticles contributes to 
the degradation [40]. These nanoparticles did not exhibit significant cytotoxicity, as evaluated using murine macrophage cells (RAW264.7). Doxorubicin loading and release were examined.
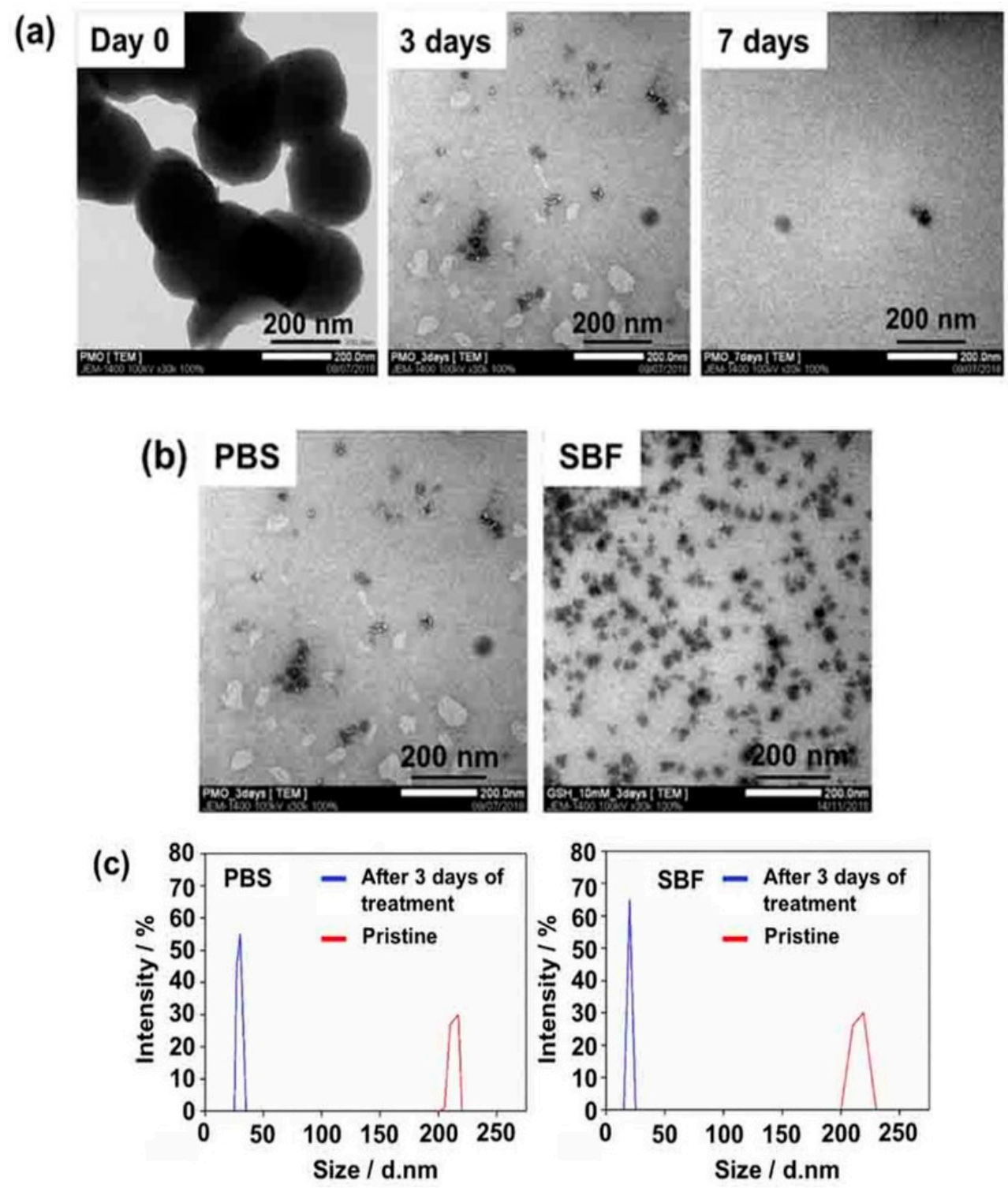

Figure 2. In vitro degradation of BPMOs: (a) TEM of degraded BPMO after incubating in PBS with glutathione (GSH) (10 mM) for various times. (b) TEM of BPMO after incubating with GSH (10 mM) in PBS or simulated body fluid (SBF) for 3 days. (c) Dynamic light scattering (DLS) measurements confirmed the average size of pristine BPMOs and degraded fragments after 3 days of treatment. Adapted from [39], Wiley Publications, 2020.

Chen et al. reported biodegradable mesoporous organosilica nanosheets for the chemotherapy $/ \mathrm{mild}$ thermotherapy of cancer with fast internalization, high cellular uptake, and high drug loading [41]. To compare these with silica-based materials, polyethylene glycol (PEG)-grafted copper monosulfide (CuS)@ mesoporous organosilica nanocapsules (MONs) are more efficient in the uptake of DOX $(859 \mu \mathrm{g} / \mathrm{mg})$. The ultra-thin porous nanosheet structure is responsible for the efficient degradation of PCMON, because $10 \mathrm{mM}$ of GSH collapsed the PCMON. According to the above results, PCMONs are excellent biodegradable materials for drug delivery. In addition, with the above experiment, thermotherapy helps in the uptake and release DOX. In conclusion, PCMONs act like triple stimulus-responsive materials ( $\mathrm{pH}$ value, GSH concentration, and laser irradiation); they accumulate in the cancer cells and destroy the tumor cells. 
Wu et al. reported a hollow mesoporous organosilica nanocapsule (HMONs) framework containing disulfide bonds. PBS medium was used to confirm the disulfide bond breaks (biodegradation) in HMONs. Three days later, after dispersing the HMONs in GSH (10 mM), the authors used TEM to confirm the structural changes in the materials. Slight changes were noticed, but, seven days later, the HMONs' shape and structure had completely collapsed. Two weeks later, the degradation was accelerated by the effect of reductive GSH-containing PBS. The hydrodynamic diameter of the materials showed decreased trends after 2 weeks. To support the above results, ICP analysis also showed a similar trend of Si presence. In conclusion, disulfide bonds containing HMONs have a high biodegradability [42].

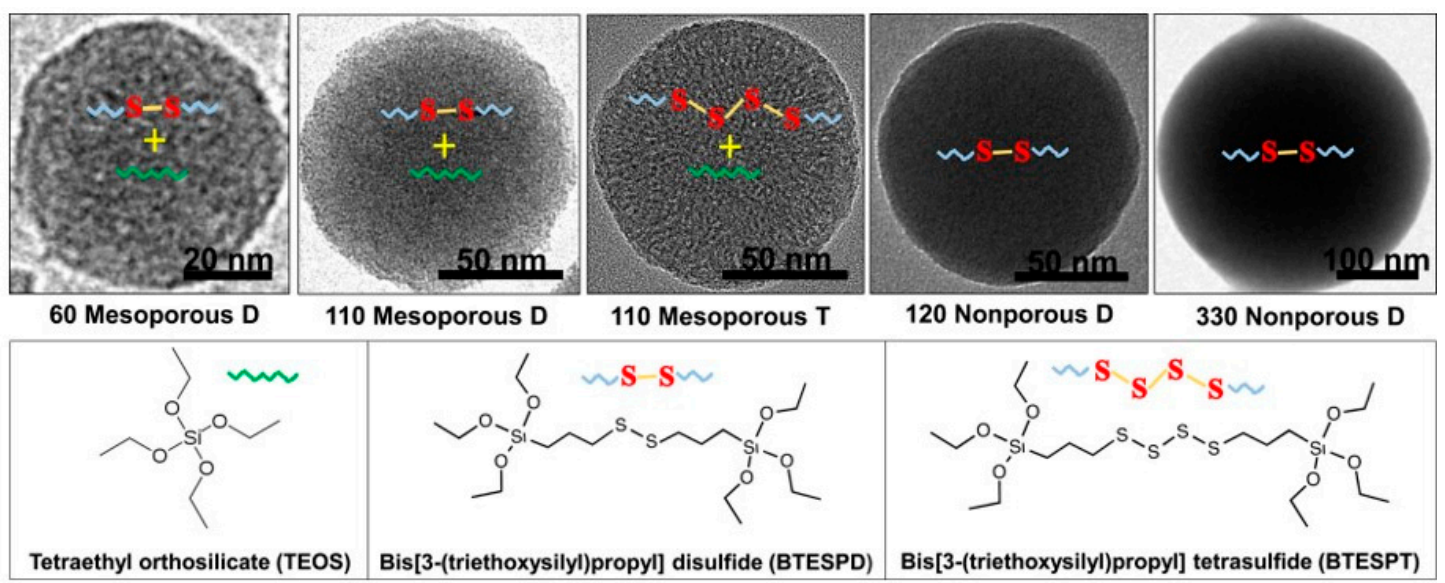

(a)

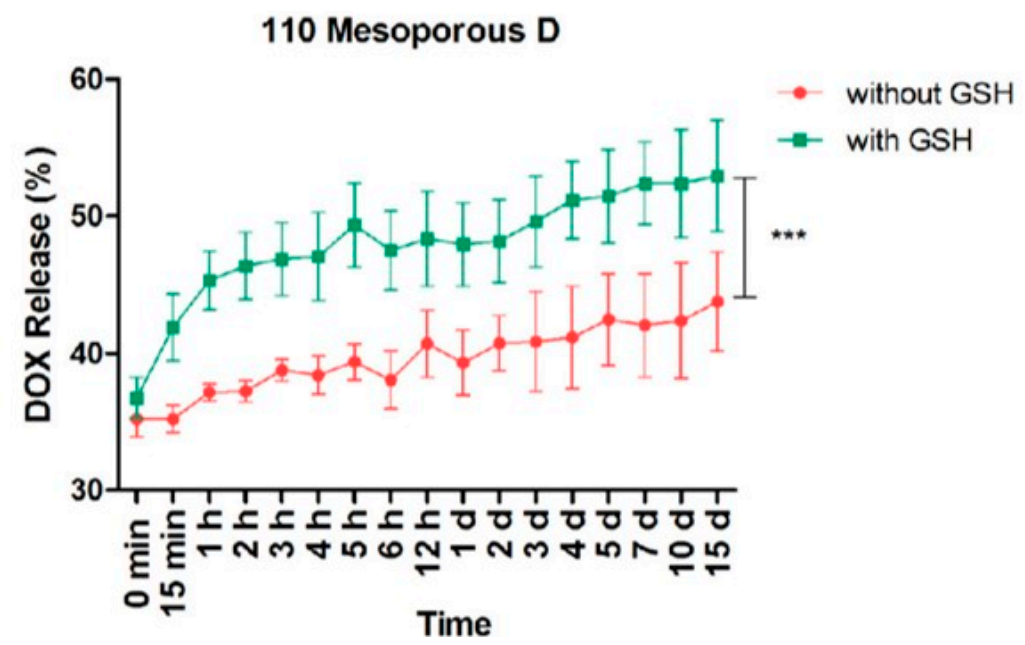

(b)

Figure 3. (a). Examples of degradable nanoparticles are prepared by Moghaddam et al. [40]. The top panel shows a transmission electron microscope (TEM) images. The bottom panel shows precursors used

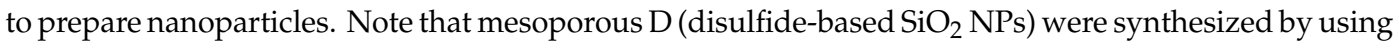
the combination of two precursors, tetraalkoxysilane (TEOS) and bis-(triethoxysilylpropyl)tetrasulfide (BTESPT), while mesoporous $\mathrm{T}$ (tetrasulfide-based $\mathrm{SiO}_{2} \mathrm{NPs}$ ) were synthesized using the combination of TEOS and BTESPT. Nanoporous D (disulfide-based $\mathrm{SiO}_{2} \mathrm{NPs}$ ) was synthesized by using TEOS. (b). Doxorubicin (DOX) release from 110 Mesoporous D nanoparticles with and without GSH. Reprinted with permission from [40], ACS Publications, 2017. 


\section{4. pH or Enzyme Responsive Systems}

Low $\mathrm{pH}$ conditions inside the endocytic pathway have been exploited for the degradation of nanomaterials and the release of the cargo, as discussed here. This approach is advantageous, as many tumor cells have lower $\mathrm{pH}$ values compared with normal cells. In addition, enzyme activities such as protease activity have been exploited for degradation.

Rao et al. prepared a cystamine-integrated periodic mesoporous organosilica (Cys-PMO). This nanoparticle possesses ordered hexagonal symmetry with a cylindrical shape and contains disulfide bonds. The loading and release of DOX were investigated, which revealed high DOX loading efficiency (50.6\%) and the release of DOX at a low $\mathrm{pH}$ (Figure 4). Thus, this system is described as a dual ( $\mathrm{pH}$ and redox)-sensitive system and appears to be promising as an anti-cancer drug for the intracellular cancer drug delivery system [43].

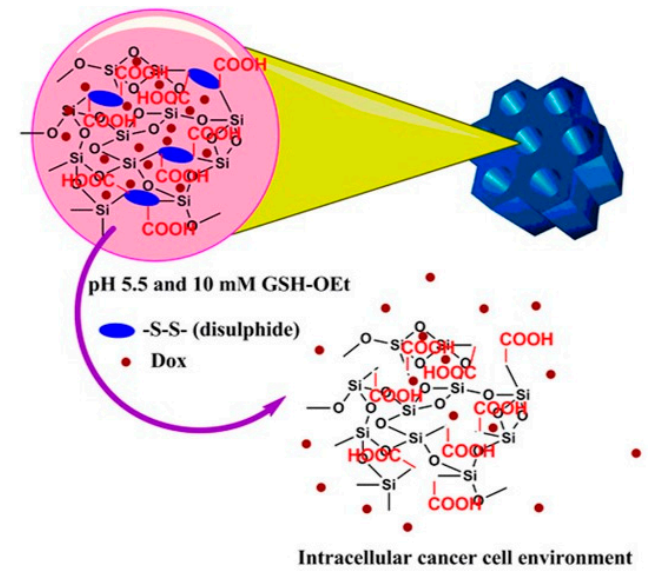

(a)

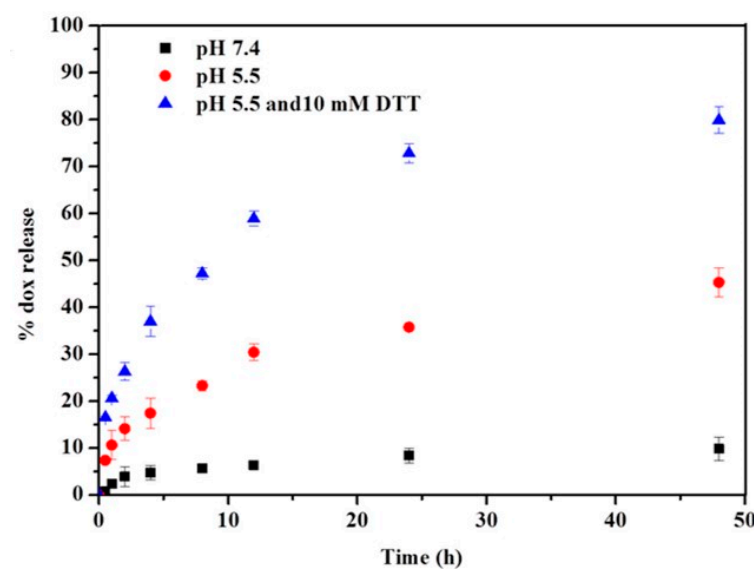

(b)

Figure 4. (a) Schematic representation of destabilization of cystamine-integrated periodic mesoporous organosilica (Cys-PMO) hybrid nanoparticles in response to redox and acidic $\mathrm{pH}$ environment and (b) in vitro DOX release profile from Cys-PMO hybrid nanoparticles. Reprinted with permission from [43], ACS Publications, 2017.

Zhang et al. synthesized PMOs that contained histidine amino acid (His-PMO) using pluronic P123 as a template. His-PMOs keep the ordered mesopores to uptake and release the drug compounds. Here, paclitaxel was used for anti-cancer drug delivery for an in vitro experiment. The high electrostatic interaction with the anti-cancer drug results in the high loading amount $(28 \mathrm{mg} / \mathrm{g})$. Finally, the results show that the release percentage and speed of paclitaxel (PTX) are completely dependent on $\mathrm{pH}$ values [44].

For controlled drug delivery applications, Motealleh et al. demonstrated PMO/alginate nanocomposite (NC) scaffolds in a pH-dependent manner. First, the anti-cancer drug was loaded into the pores and then the biopolymer coating was done. Later, the PMOs are embedded into an alginate network. The $\mathrm{pH}$ plays a major role in releasing the anti-cancer drug from the pores. In conclusion, the non-biopolymer-coated scaffold had a higher efficiency in delivering the drug to the cells in comparison with the biopolymer coating. However, the biopolymer coated scaffold was useful for slow and prolonged drug release [45]. Later, Motealleh et al. prepared self-assembled monolayers (SAMs) of pH-responsive chiral PMOs for drug delivery ability [46].

Moorthy et al. synthesized safranin-diurea-bridged hybrid mesoporous organosilica (SDU- HMS) for simultaneous diagnosis and therapy. The anti-cancer drug 5-fluorouracil (5-FU) was loaded by the interactions between the drug molecule and the NH-CO-NH part of the diurea ligands that exist in the pore walls. The drug release was low-pH dependent. The safranin fluorophore provided stable fluorescence in the nanoparticle. These experimental results proved that the SDU-HMS nanocarrier is 
desirable for red fluorescence-based in vitro tracking and $\mathrm{pH}$-responsive anti-cancer drug release in cancer therapy [47].

Omar et al. used diazobenzene-triethoxysilyl amide precursors to synthesize BPMO nanoparticles that contained azobenzene linkers. Condensation was carried out with aromatic benzene or aliphatic ethane to produce an AZO-B (azobenzene linkers were condensed with aromatic benzene) or AZO-E (azobenzene linkers were condensed with aliphatic ethane) framework. This enabled the evaluation of the framework structure in relation to biodegradation, doxorubicin loading, and release. The authors showed that closer pore packing in AZO-B enhanced the enzymatic biodegradation of these hybrid frameworks compared with AZO-E. The above results show that a self-assembly pattern will be a useful drug delivery system in future [48]. The degradation of AZO-B and AZO-E can be catalyzed by azoreductase, which is known to cleave the azo-bond. This may have significance for colon-specific drug delivery.

Croissant et al. synthesized oxamide phenylene-based BPMO nanoparticles. The oxamide function provided biodegradability in the presence of trypsin that was used as a model enzyme. This system had excellent drug loading efficiency and was efficient in drug delivery to cancer cells [49].

Lu et al. reported yolk-shell structure-based PMOs for cancer cell targeting and drug delivery, which contained disulfide bonds. Near-infrared fluorescence (NIRF) dye was used on the surface of the materials with anti-Her2 affibody to target the cancer cells. Disulfide-containing PMOs are good candidates to deliver the anti-cancer drug DOX due to the GSH-dependent release. The above results indicate that PMOs are used for NIR Imaging, GSH, and $\mathrm{pH}$-responsive drug release with cancer-targeting properties [50]. One year later, $\mathrm{Lu}$ et al. developed copper sulfide PMOs for hyperthermia-enhanced chemotherapy because of the photothermal effect of CuS. In addition, this material is effective for drug release in response to GSH, $\mathrm{pH}$, and laser irradiation [51].

Moorthy et al. reported pyridine-modified PMOs for cancer therapy, which are called disilylated 2,6-dimethylpyridine-PMOs (DMPy-PMOs). They have enough efficiency to kill the cancer cells after 5-FU loading into the pores without toxicity. PMO materials have more efficiency in carrying drugs. They have the efficiency to kill cancer cells that depend on the $\mathrm{pH}$ and concentration of the materials. DMPy-PMOs were used due to their good absorbent properties in relation to removing metal ions [52].

Daurat et al. reported organosilica nanoparticles with either amine or ammonium walls constituting their structure. These nanoparticles are special in the sense that they can store and deliver gemcitabine or gemcitabine monophosphate (GMP), hydrophilic anti-cancer drugs that cannot be encapsulated in MSNs without using a pore cap. The release of GMP was dependent on a low $\mathrm{pH}$. The nanoparticles were endocytosed by MCF-7 breast cancer cells and were efficient in causing cancer cell death [53].

\section{Light and Ultrasound Responsive Systems}

Shao et al. synthesized $\mathrm{MoS}_{2}$ nanosheet-covered PMOs (326 nm) with PEG decoration for NIR light-responsive drug delivery. The above materials uptake high-level DOX (160 $\left.\mu \mathrm{g} \cdot \mathrm{mg}^{-1} \mathrm{PMOs}\right)$ into the pores and have an excellent dispersion in physiological conditions. Upon light triggering, the materials have an excellent photothermal effect. Laser light of $808 \mathrm{~nm}$ breaks the $\mathrm{MOS}_{2}$ structure due to the photothermal effect, and drug release from the materials then occurs. The authors used two types of cancer cells (liver and breast) for combined photothermal therapy and chemotherapy. The final result showed more efficiency compared with chemotherapy or photothermal therapy alone [54].

Croissant et al. used PMO materials with an ethynylene bridge for photodynamic therapy and two-photon imaging applications. Here, a large two-photon absorber with four trimethylsilyl groups was used to capture NIR light. In addition, the PMO materials contained azidopropyltriethoxysilane in their structure. Later, the photosensitizer (propargylated fluorescent bromo-quinoline) was attached by click chemistry to generate singlet oxygen. After investigating the two-photon properties of the PMOs, the authors confirmed the fluorescence resonance energy transfer (FRET) from the fluorophore to the photosensitizer. The MCF-7 cell lines showed efficient cell death after irradiation with two-photon light $[27,29]$. 
Jimenez et al. prepared nanodiamond-encapsulated PMO, then anti-cancer drug doxorubicin was loaded in the pores. The drug release was dependent on $\mathrm{pH}$. Later, two-photon exposure was used to generate singlet oxygen species. The above system worked in two ways to kill the cancer cells incubated with DOX-loaded ND@PMO [55].

Lin et al. developed protoporphyrin IX-encapsulated PMOs for light-induced metabolic inactivation of tumor cells. The synthesis involved the modification of the hydrophobic benzene group in the PMO framework by installing the hydroxyl group to incorporate photosensitizers. The above studies showed that the design of the post-modified nanocomposite was highly advantageous for in vitro photodynamic therapy (PDT) in human colon cancer cells (Figure 5) [56].

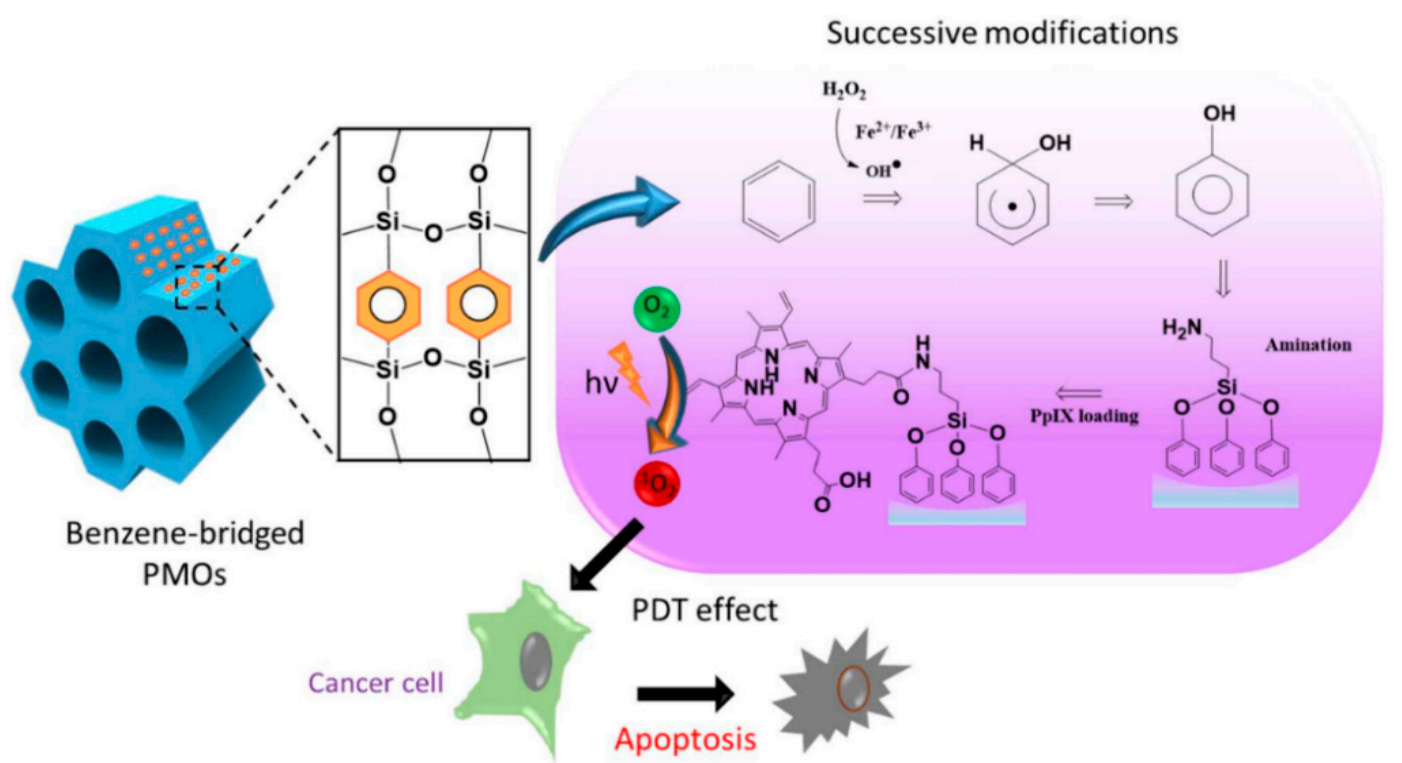

Figure 5. The reaction pathway of the hydroxylation of benzene and protoporphyrin IX loading and the mechanism elucidating the light-induced biological effects (Apoptosis). Reprinted from [56], MDPI Publications, 2020.

Wu et al. synthesized multifunctional PMO-DOX@MoS 2 -Polyethylenimine (PEI) - Bovine serum albumin (BSA)- Folic acid (FA) by wrapping $\mathrm{MoS}_{2}$ nanosheets onto DOX-loaded thioether-bridged PMO (PMO-SH) and then modifying them with the targeting moiety BSA-FA. The prepared PMO-DOX@MoS - -PEI-BSA-FA had a uniform diameter $(196 \mathrm{~nm})$ and high drug-loading capacity (185 mg.g ${ }^{-1}$ PMO-SH). Due to the excellent photothermal properties of $\mathrm{MoS}_{2}$ nanosheets, NIR laser irradiation resulted in the generation of heat, causing release of DOX in vitro and in vivo (Figure 6) [57].

Qian et al. reported hybrid hollow PMOs (HPMOs) for combined cancer therapy-ultrasound combined with chemotherapy. Because the hollow nanostructure enhances the ultrasound efficiency to change the acoustic microenvironment of the tissues, it received a large ultrasound energy deposition. The hollow sphere area was used as a reservoir for anti-cancer drug storage; then, drugs were released because of the ultrasound irradiation. The specific framework induced $\mathrm{p}-\mathrm{p}$ supramolecular stacking between the benzene group-bridged framework and doxorubicin molecules. In addition, within the above experiment, the authors used HPMOs for cancer therapy in a mouse xenograft model. This system also shows good results in developing novel cancer therapy.

In Table 1, we summarize the PMOs and organo bridges discussed in this review for drug delivery applications. In addition, we also list the anti-cancer drug release systems using internal or external stimuli. 
Table 1. PMOs for drug delivery with internal and external stimuli.

\begin{tabular}{|c|c|c|c|c|c|c|}
\hline PMOs & Organo Bridge & Drug & Loading Efficiency & Biodegradation/Stimulus & Experiment & Reference \\
\hline Nanorods and Nanosphere & Ethylene-bis(propyl)disulfide & Doxorubicin & $22.20 \mathrm{wt} \%$ & $\mathrm{pH}$ & in vitro & [13] \\
\hline Nanoparticles & ethylene-azidopropyl & Doxorubicin & $14.20 \mathrm{wt} \%$ & $\mathrm{pH}$ and Two-photon & in vitro & [29] \\
\hline Biodegradable PMO & $\begin{array}{c}\text { bis [3-(triethoxysilyl) propyl] } \\
\text { tetrasulfide }\end{array}$ & Daunorubicin & $12.04 \mathrm{wt} \%$ & GSH & in vivo & [39] \\
\hline Cys PMO & Disulfide & Doxorubicin & $50.60 \mathrm{wt} \%$ & $\mathrm{pH}, \mathrm{GSH}$ & in vitro & [43] \\
\hline $\mathrm{PMO}-\mathrm{NH}_{2}$ & 1,2-bis(trimethoxysilyl)ethane & Doxorubicin & $88.60 w t \%$ & $\mathrm{pH}$ & in vitro & [45] \\
\hline Yolk-shell PMOs & Thioether & Doxorubicin & $31.70 w t \%$ & GSH & in vivo & {$[50]$} \\
\hline CuS@PMOs & $\begin{array}{l}\text { 1,4-Bis(triethoxysily)propane } \\
\text { tetrasulfide }\end{array}$ & Doxorubicin & $94.00 w t^{\circ} \%$ & GSH & in vivo & [51] \\
\hline DMPy-PMO & N,N'-Disilylated Pyridine & 5-Fluorouracil & $128 \mathrm{mg} \cdot \mathrm{g}^{-1}$ & $\mathrm{pH}$ & in vitro & [52] \\
\hline Nanoparticles & Benzene & Protoporphyrin IX & $10.80 \mathrm{wt} \%$ & green laser light at $532 \mathrm{~nm}$ & in vitro & [56] \\
\hline $\mathrm{MoS}_{2}$ nanosheet-capped PMOs & Thioether & Doxorubicin & $185 \mathrm{mg} \cdot \mathrm{g}^{-1}$ & $\mathrm{pH}$ and NIR Light & in vivo & [57] \\
\hline Hollow PMOs & Benzene & Doxorubicin & $369 \mathrm{mg} \cdot \mathrm{g}^{-1}$ & ultrasound & in vivo & [58] \\
\hline
\end{tabular}




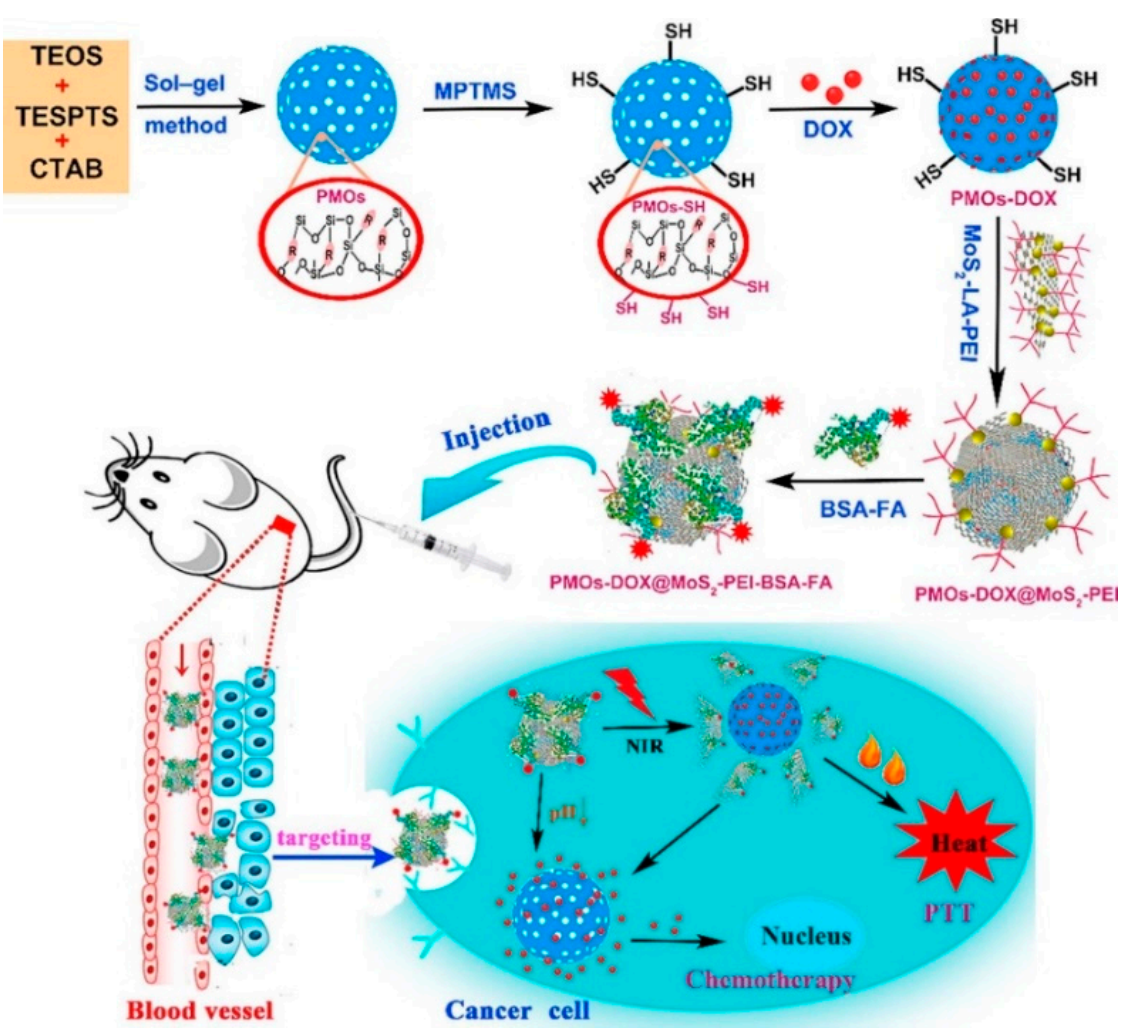

Figure 6. Schematic illustration for the synthesis and preparation of PMO-DOX@MoS 2 - lipoic acid (LA)-PEI-BSA-FA composite as a multifunctional drug delivery system for synergistic chemo-photothermal targeted therapy of tumors. Reprinted with permission from [57], Elsevier Publications, 2018.

\section{Related Studies}

Thiol organosilica NPs represent a type of silica nanoparticles that are synthesized by the sol-gel method using a thiol organosilicate as a precursor. These nonporous silica nanoparticles are synthesized by using a precursor, 3-mercaptopropyltrimethoxysilane (MPMS). Doura et al. demonstrated that the use of a different precursor, 3-mercaptopropyl(dimethoxy)methylsilane (MPDMS), results in the preparation of nanoparticles that contain abundant disulfide bonds, as revealed by nuclear magnetic resonance (NMR) measurements and Raman spectroscopy [59]. They also used the combination of MPMS and MPDMS, which revealed that the ratio of disulfide bonds to thiols increased with the increase in MPDMS. The formation of disulfide bonds in the nanoparticles synthesized using MPDMS is due to their chemical composition, which enables the maximum number of two-siloxane bonds to be formed. Therefore, nanoparticles are formed only when disulfide bonds are formed within the framework. The degradation of nanoparticles containing disulfide bonds after incubation with $10 \mathrm{mM}$ GSH for 7 days was confirmed using TEM analyses. A high ratio of MPDMS gives more degradation compared with free MPMS NPs.

Later, Mekaru et al. evaluated the biodegradability of disulfide-organosilica nanoparticles by soft x-ray photoelectron spectroscopy for cancer therapy implications [60]. They used two kinds of organosilica nanoparticles, MPMS and MPDMS, that were fabricated similarly to those described by Doura et al. [59]. Disulfide bonds are easily degraded by incubating with $10 \mathrm{mM} \mathrm{GSH}$ concentration at $37^{\circ} \mathrm{C}$ for 7 days. The SEM analysis of MPDMS NPs showed biodegradation after 7 days, while MPMS NPs were not degraded. The soft X-ray photoelectron spectroscopy results of MPDMS before and after GSH treatment were consistent with the degradation of disulfide bonds by GSH. These results support the knowledge that MPDMS NPs possess a biodegradable feature that is advantageous for clinical translation in nanomedicine. 


\section{Summary and Future Prospects}

PMOs contain organic-inorganic hybrid materials with highly ordered structures, uniform pore sizes, and a homogenous distribution of organic bridges in a silica framework. The addition of an organic bridge disrupts the periodic ordering of mesopores and monodisperse PMOs and expands the application of silica-based nanomaterials so that broad applications are possible.

Of particular importance are BPMOs that contain biodegradable bonds, including redox-sensitive bonds, $\mathrm{pH}$-sensitive bonds, and bonds that are sensitive to enzymatic activities. A variety of studies have been carried out with BPMOs containing redox-sensitive bonds such as disulfide bonds and tetrasulfide bonds. The degradation of these nanoparticles under redox conditions was much faster compared to mesoporous silica nanoparticles without redox-sensitive bonds. In addition, the degradation of nonporous nanoparticles was slower, pointing to the importance of the mesoporous structure. Studies that demonstrate the in vivo degradation of BPMOs are limited, and this issue needs to be extensively investigated in the future, including excretion studies of BPMOs from an animal body. While this manuscript was being reviewed, a review paper discussing trends in degradable mesoporous organosilica-based nanomaterials for controlling drug delivery was published [61]. That review contains even more discussion on nonporous materials.

BPMOs have been shown to be effective in loading anti-cancer drugs and accomplishing the controlled release of these drugs. In this sense, it is important to point out that the presence of an organic moiety in BPMOs contributes to the loading of cargos to the nanoparticle due to the occurrence of additional chemical interactions between the cargo and the delivery vehicle. Furthermore, the release of the drugs can be carried out in a controlled manner, such as under reducing conditions, low-pH conditions, etc. Light-responsive release can also be carried out. This realization led to the development of various stimuli-responsive systems based on BPMOs.

Author Contributions: S.C. and F.T. wrote this manuscript. All authors have read and agreed to the published version of the manuscript.

Funding: This work was funded by japan society for the promotion of science (JSPS) Grants-in-Aid for Scientific Research (KAKENHI), grant numbers JP15K21764 and JP20H00331.

Acknowledgments: We would like to thank members of the Tamanoi Lab for the valuable discussions we had.

Conflicts of Interest: The authors declare no conflict of interest.

$\begin{array}{ll}\text { Abbreviations } \\ \text { PMOs } & \text { periodic mesoporous organosilicas } \\ \text { BPMOs } & \begin{array}{l}\text { biodegradable periodic mesoporous organosilica nanoparticles } \\ \text { enhanced permeability and retention }\end{array} \\ \text { EPR } & \text { Mesoporous silica nanoparticles } \\ \text { MSNs } & \text { cetyltrimethylammonium bromide } \\ \text { CTAB } & \text { tetraalkoxysilane } \\ \text { TEOS } & \text { hydrodynamic diameter } \\ \text { HD } & \text { glutathione } \\ \text { GSH } & \text { Ethylene-Bis(Propyl) Disulfide } \\ \text { EDIS } & \text { Mixed PMO } \\ \text { MPMO } & \text { Doxorubicin } \\ \text { DOX } & \text { Chick Chorioallantoic Membrane } \\ \text { CAM } & \text { Phosphate-buffered saline } \\ \text { PBS } & \text { simulated body fluid } \\ \text { SBF } & \text { Daunorubicin } \\ \text { DNR } & \text { transmission electron microscope } \\ \text { TEM } & \text { bis-(triethoxysilylpropyl)tetrasulfide } \\ \text { BTESPT } & \text { Mesoporous Organosilica Nanosheets } \\ \text { PCMONSs } & \text { hollow mesoporous organosilica nanocapsule } \\ \text { HMONs } & \text { Inductively coupled plasma } \\ \text { ICP } & \end{array}$




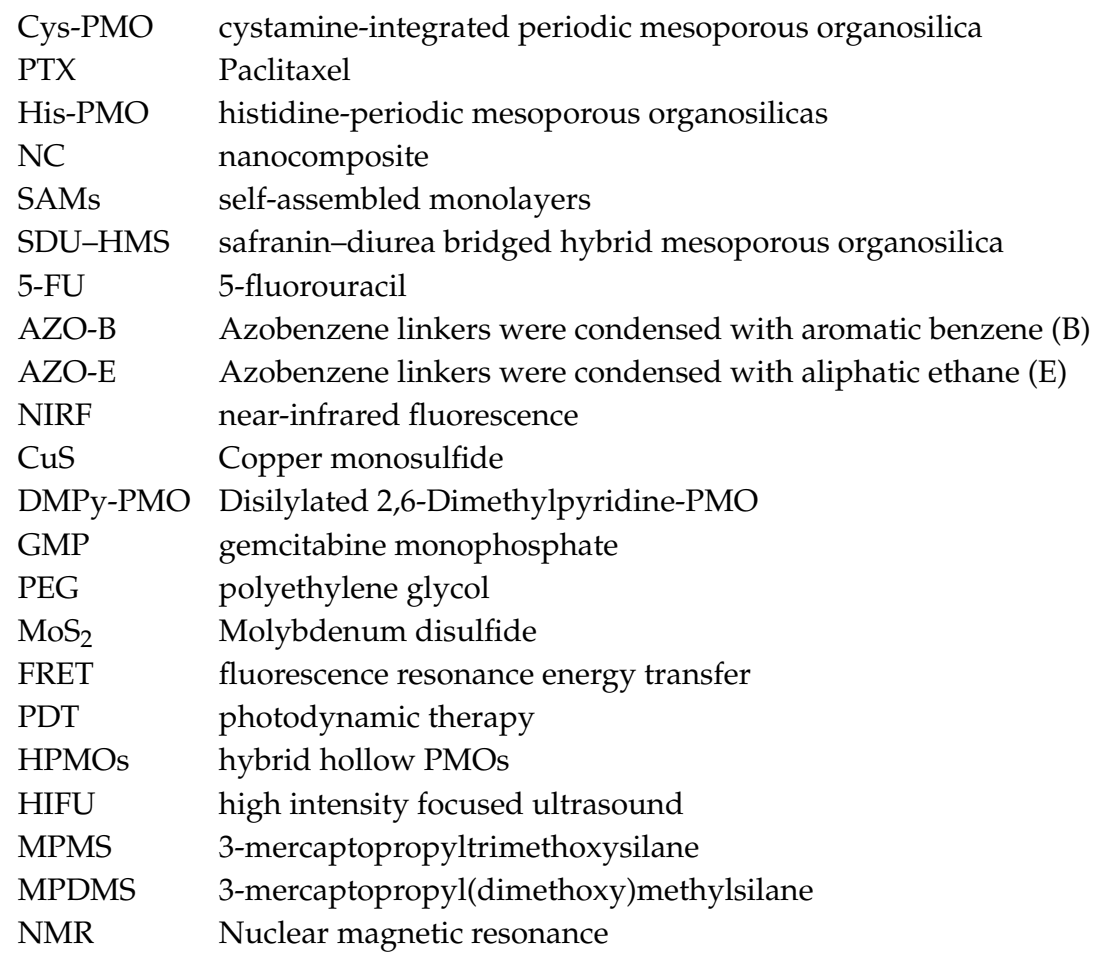

\section{References}

1. Siegel, R.L.; Miller, K.D.; Jemal, A. Cancer statistics, 2020. CA Cancer J. Clin. 2020, 70, 7-30. [CrossRef]

2. Shi, Y.; van der Meel, R.; Chen, X.; Lammers, T. The EPR effect and beyond: Strategies to improve tumor targeting and cancer nanomedicine treatment efficacy. Theranostics 2020, 10, 7921-7924. [CrossRef]

3. Nehoff, H.; Parayath, N.N.; Domanovitch, L.; Taurin, S.; Greish, K. Nanomedicine for drug targeting: Strategies beyond the enhanced permeability and retention effect. Int. J. Nanomed. 2014, 9, 2539-2555. [CrossRef]

4. $\quad$ Karthick, V.; Panda, S.; Kumar, V.G.; Kumar, D.; Shrestha, L.K.; Ariga, K.; Vasanth, K.; Chinnathambi, S.; Dhas, T.S.; Suganya, K.S.U. Quercetin loaded PLGA microspheres induce apoptosis in breast cancer cells. Appl. Surf. Sci. 2019, 487, 211-217. [CrossRef]

5. Chinnathambi, S.; Shirahata, N. Recent advances on fluorescent biomarkers of near-infrared quantum dots for in vitro and in vivo imaging. Sci. Technol. Adv. Mater. 2019, 20, 337-355. [CrossRef]

6. Chinnathambi, S.; Chen, S.; Ganesan, S.; Hanagata, N. Binding mode of CpG oligodeoxynucleotides to nanoparticles regulates bifurcated cytokine induction via Toll-like receptor 9. Sci. Rep. 2012, 2, 534. [CrossRef]

7. Ha, C.-S.; Park, S.S. PMOs as hosts for drug and biomolecules. In Periodic Mesoporous Organosilicas; Springer: Singapore, 2019; pp. 189-218. [CrossRef]

8. Moorthy, M.S.; Park, S.-S.; Fuping, D.; Hong, S.-H.; Selvaraj, M.; Ha, C.-S. Step-up synthesis of amidoxime-functionalised periodic mesoporous organosilicas with an amphoteric ligand in the framework for drug delivery. J. Mater. Chem. 2012, 22, 9100. [CrossRef]

9. Esquivel, D.; Van Der Voort, P.; Romero-Salguero, F. Designing advanced functional periodic mesoporous organosilicas for biomedical applications. AIMS Mater. Sci. 2014, 1, 70-86. [CrossRef]

10. Du, X.; Kleitz, F.; Li, X.; Huang, H.; Zhang, X.; Qiao, S.-Z. Disulfide-bridged organosilica frameworks: Designed, synthesis, redox-triggered biodegradation, and nanobiomedical applications. Adv. Funct. Mater. 2018, 28, 1707325. [CrossRef]

11. Kresge, C.T.; Leonowicz, M.E.; Roth, W.J.; Vartuli, J.C.; Beck, J.S. Ordered mesoporous molecular sieves synthesized by a liquidcrystal template mechanism. Nature 1992, 359, 710-712.

12. Prasetyanto, E.A.; Bertucci, A.; Septiadi, D.; Corradini, R.; Castro-Hartmann, P.; De Cola, L. Breakable hybrid organosilica nanocapsules for protein delivery. Angew. Chem. Int. Ed. Engl. 2016, 55, 3323-3327. [CrossRef]

13. Croissant, J.; Cattoen, X.; Man, M.W.; Gallud, A.; Raehm, L.; Trens, P.; Maynadier, M.; Durand, J.O. Biodegradable ethylene-bis(propyl)disulfide-based periodic mesoporous organosilica nanorods and nanospheres for efficient in-vitro drug delivery. Adv. Mater. 2014, 26, 6174-6180. [CrossRef] 
14. Vallet-Regi, M.; Tamanoi, F. Overview of studies regarding mesoporous silica nanomaterials and their biomedical application. Enzymes 2018, 43, 1-10. [CrossRef]

15. Croissant, J.G.; Brinker, C.J. Biodegradable silica-based nanoparticles: Dissolution kinetics and selective bond cleavage. Enzymes 2018, 43, 181-214. [CrossRef]

16. Croissant, J.G.; Fatieiev, Y.; Khashab, N.M. Degradability and clearance of silicon, organosilica, silsesquioxane, silica mixed oxide, and mesoporous silica nanoparticles. Adv. Mater. 2017, 29, 1604634. [CrossRef]

17. Wang, W.; Lofgreen, J.E.; Ozin, G.A. Why PMO? Towards functionality and utility of periodic mesoporous organosilicas. Small 2010, 6, 2634-2642. [CrossRef]

18. Hatton, B.; Landskron, K.; Whitnall, W.; Perovic, D.; Ozin, G.A. Past, present, and future of periodic mesoporous organosilicass the PMOs. Acc. Chem. Res. 2005, 38, 305-312.

19. Hunks, W.J.; Ozin, G.A. Challenges and advances in the chemistry of periodic mesoporous organosilicas (PMOs). J. Mater. Chem. 2005, 15, 3716. [CrossRef]

20. Cho, E.-B.; Park, J.; Jaroniec, M. Structural stability of Si-C bonds in periodic mesoporous thiophene-silicas prepared under acidic conditions. J. Phys. Chem. C 2013, 117, 21441-21449. [CrossRef]

21. Van der Voort, P.; Esquivel, D.; De Canck, E.; Goethals, F.; Van Driessche, I.; Romero-salguero, F.J. Periodic mesoporous organosilicas: From simple to complex bridges; a comprehensive overview of functions, morphologies and applications. Chem. Soc. Rev. 2013, 42, 3913-3955. [CrossRef]

22. Hoffmann, F.; Cornelius, M.; Morell, J.; Froba, M. Periodic mesoporous organosilicas (PMOs): Past, present, and future. J. Nanosci. Nanotechnol. 2006, 6, 265-288. [CrossRef]

23. Guan, B.; Cui, Y.; Ren, Z.; Qiao, Z.A.; Wang, L.; Liu, Y.; Huo, Q. Highly ordered periodic mesoporous organosilica nanoparticles with controllable pore structures. Nanoscale 2012, 4, 6588-6596. [CrossRef]

24. Park, S.S.; Santha Moorthy, M.; Ha, C.-S. Periodic mesoporous organosilicas for advanced applications. NPG Asia Mater. 2014, 6, e96. [CrossRef]

25. Kaczmarek, A.M.; Van Der Voort, P. Light-emitting lanthanide periodic mesoporous organosilica (PMO) hybrid materials. Materials 2020, 13, 566. [CrossRef]

26. Croissant, J.G.; Cattoen, X.; Wong, M.C.; Durand, J.O.; Khashab, N.M. Syntheses and applications of periodic mesoporous organosilica nanoparticles. Nanoscale 2015, 7, 20318-20334. [CrossRef]

27. Croissant, J.G.; Fatieiev, Y.; Almalik, A.; Khashab, N.M. Mesoporous silica and organosilica nanoparticles: Physical chemistry, biosafety, delivery strategies, and biomedical applications. Adv. Healthc. Mater. 2018, 7, 1700831. [CrossRef]

28. Du, X.; Li, X.; Xiong, L.; Zhang, X.; Kleitz, F.; Qiao, S.Z. Mesoporous silica nanoparticles with organo-bridged silsesquioxane framework as innovative platforms for bioimaging and therapeutic agent delivery. Biomaterials 2016, 91, 90-127. [CrossRef]

29. Croissant, J.G.; Picard, S.; Aggad, D.; Klausen, M.; Mauriello Jimenez, C.; Maynadier, M.; Mongin, O.; Clermont, G.; Genin, E.; Cattoen, X.; et al. Fluorescent periodic mesoporous organosilica nanoparticles dual-functionalized via click chemistry for two-photon photodynamic therapy in cells. J. Mater. Chem. B 2016, 4, 5567-5574. [CrossRef]

30. Guan, M.; Wang, W.; Henderson, E.J.; Dag, O.; Kubel, C.; Chakravadhanula, V.S.; Rinck, J.; Moudrakovski, I.L.; Thomson, J.; McDowell, J.; et al. Assembling photoluminescent silicon nanocrystals into periodic mesoporous organosilica. J. Am. Chem. Soc. 2012, 134, 8439-8446. [CrossRef]

31. Li, H.; Raehm, L.; Charnay, C.; Durand, J.O.; Pleixats, R. Preparation and characterization of novel mixed periodic mesoporous organosilica nanoparticles. Materials 2020, 13, 1569. [CrossRef]

32. Ha, C.-S.; Park, S.S. Synthetic routes and new precursors for the preparation of PMOs. In Periodic Mesoporous Organosilicas; Springer: Singapore, 2019; pp. 87-100. [CrossRef]

33. Vercaemst, C.; Ide, M.; Wiper, P.V.; Jones, J.T.A.; Khimyak, Y.Z.; Verpoort, F.; Van Der Voort, P. Ethenylene-bridged periodic mesoporous organosilicas: FromEtoZ. Chem. Mater. 2009, 21, 5792-5800. [CrossRef]

34. Asefa, T.; MacLachlan, M.J.; Coombs, N.; Ozin, G.A. Periodic mesoporous organosilicas with organic groups inside the channel walls. Nature 1999, 402, 867-871.

35. Vert, M.; Doi, Y.; Hellwich, K.-H.; Hess, M.; Hodge, P.; Kubisa, P.; Rinaudo, M.; Schué, F. Terminology for biorelated polymers and applications (IUPAC Recommendations 2012). Pure Appl. Chem. 2012, 84, 377-410. [CrossRef]

36. Maggini, L.; Cabrera, I.; Ruiz-Carretero, A.; Prasetyanto, E.A.; Robinet, E.; De Cola, L. Breakable mesoporous silica nanoparticles for targeted drug delivery. Nanoscale 2016, 8, 7240-7247. [CrossRef] 
37. Liu, J.; Yu, M.; Zhou, C.; Zheng, J. Renal clearable inorganic nanoparticles: A new frontier of bionanotechnology. Mater. Today 2013, 16, 477-486. [CrossRef]

38. Vu, B.T.; Shahin, S.A.; Croissant, J.; Fatieiev, Y.; Matsumoto, K.; Le-Hoang Doan, T.; Yik, T.; Simargi, S.; Conteras, A.; Ratliff, L.; et al. Chick chorioallantoic membrane assay as an in vivo model to study the effect of nanoparticle-based anti-cancer drugs in ovarian cancer. Sci. Rep. 2018, 8, 8524. [CrossRef]

39. Mai, N.X.D.; Birault, A.; Matsumoto, K.; Ta, H.K.T.; Intasa-Ard, S.G.; Morrison, K.; Thang, P.B.; Doan, T.L.H.; Tamanoi, F. Biodegradable periodic mesoporous organosilica (BPMO) loaded with daunorubicin: A promising nanoparticle-based anticancer drug. ChemMedChem 2020, 15, 593-599. [CrossRef]

40. Moghaddam, S.P.H.; Saikia, J.; Yazdimamaghani, M.; Ghandehari, H. Redox-responsive polysulfide-based biodegradable organosilica nanoparticles for delivery of bioactive agents. ACS Appl. Mater. Interfaces 2017, 9, 21133-21146. [CrossRef]

41. Chen, L.; Meng, X.; Liu, M.; Lv, R.; Cai, B.; Wang, Z. Biodegradable mesoporous organosilica nanosheets for chemotherapy/mild thermotherapy of cancer: Fast internalization, high cellular uptake, and high drug loading. ACS Appl. Mater. Interfaces 2020, 12, 30234-30246. [CrossRef]

42. Wu, J.; Bremner, D.H.; Niu, S.; Shi, M.; Wang, H.; Tang, R.; Zhu, L.M. Chemodrug-gated biodegradable hollow mesoporous organosilica nanotheranostics for multimodal imaging-guided low-temperature photothermal therapy/chemotherapy of cancer. ACS Appl. Mater. Interfaces 2018, 10, 42115-42126. [CrossRef]

43. Rao, K.M.; Parambadath, S.; Kumar, A.; Ha, C.-S.; Han, S.S. Tunable intracellular degradable periodic mesoporous organosilica hybrid nanoparticles for doxorubicin drug delivery in cancer cells. ACS Biomater. Sci. Eng. 2017, 4, 175-183. [CrossRef]

44. Zhang, W.; Ma, H.; Hua, J.; Zhang, W.; Guo, C.; Wang, J. Construction of $\mathrm{pH}$ responsive periodic mesoporous organosilica with histidine framework (His-PMO) for drug delivery. J. Solid State Chem. 2019, 277, 761-768. [CrossRef]

45. Motealleh, A.; De Marco, R.; Kehr, N.S. Stimuli-responsive local drug molecule delivery to adhered cells in a 3D nanocomposite scaffold. J. Mater. Chem. B 2019, 7, 3716-3723. [CrossRef]

46. Motealleh, A.; Dorri, P.; Kehr, N.S. Self-assembled monolayers of chiral periodic mesoporous organosilica as a stimuli responsive local drug delivery system. J. Mater. Chem. B 2019, 7, 2362-2371. [CrossRef]

47. Moorthy, M.S.; Song, H.-J.; Bae, J.-H.; Kim, S.-H.; Ha, C.-S. Red fluorescent hybrid mesoporous organosilicas for simultaneous cell imaging and anti-cancer drug delivery. RSC Adv. 2014, 4, 43342-43345. [CrossRef]

48. Omar, H.; Moosa, B.; Alamoudi, K.; Anjum, D.H.; Emwas, A.H.; El Tall, O.; Vu, B.; Tamanoi, F.; AlMalik, A.; Khashab, N.M. Impact of pore-walls ligand assembly on the biodegradation of mesoporous organosilica nanoparticles for controlled drug delivery. ACS Omega 2018, 3, 5195-5201. [CrossRef]

49. Croissant, J.C.; Fatieiev, Y.; Julfakyan, K.; Lu, J.; Emwas, A.H.; Anjum, D.H.; Omar, H.; Tamanoi, F.; Zink, J.I.; Khashab, N.M. Biodegradable oxamide-phenylene-based mesoporous organosilica nanoparticles with unprecedented drug payloads for delivery in cells. Chem. Eur. J. 2016, 22, 14806-14811. [CrossRef]

50. Lu, N.; Tian, Y.; Tian, W.; Huang, P.; Liu, Y.; Tang, Y.; Wang, C.; Wang, S.; Su, Y.; Zhang, Y.; et al. Smart cancer cell targeting imaging and drug delivery system by systematically engineering periodic mesoporous organosilica nanoparticles. ACS Appl. Mater. Interfaces 2016, 8, 2985-2993. [CrossRef]

51. Lu, N.; Huang, P.; Fan, W.; Wang, Z.; Liu, Y.; Wang, S.; Zhang, G.; Hu, J.; Liu, W.; Niu, G.; et al. Tri-stimuli-responsive biodegradable theranostics for mild hyperthermia enhanced chemotherapy. Biomaterials 2017, 126, 39-48. [CrossRef]

52. Moorthy, M.S.; Kim, M.-J.; Bae, J.-H.; Park, S.S.; Saravanan, N.; Kim, S.-H.; Ha, C.-S. Multifunctional periodic mesoporous organosilicas for biomolecule recognition, biomedical applications in cancer therapy, and metal adsorption. Eur. J. Inorg. Chem. 2013, 2013, 3028-3038. [CrossRef]

53. Daurat, M.; Rahmani, S.; Bouchal, R.; Akrout, A.; Budimir, J.; Nguyen, C.; Charnay, C.; Guari, Y.; Richeter, S.; Raehm, L.; et al. Organosilica nanoparticles for gemcitabine monophosphate delivery in cancer cells. ChemNanoMat 2019, 5, 888-896. [CrossRef]

54. Shao, T.; Wen, J.; Zhang, Q.; Zhou, Y.; Liu, L.; Yuwen, L.; Tian, Y.; Zhang, Y.; Tian, W.; Su, Y.; et al. NIR photoresponsive drug delivery and synergistic chemo-photothermal therapy by monodispersed-MoS2-nanosheets wrapped periodic mesoporous organosilicas. J. Mater. Chem. B 2016, 4, 7708-7717. [CrossRef]

55. Jimenez, C.M.; Knezevic, N.Z.; Rubio, Y.G.; Szunerits, S.; Boukherroub, R.; Teodorescu, F.; Croissant, J.G.; Hocine, O.; Seric, M.; Raehm, L.; et al. Nanodiamond-PMO for two-photon PDT and drug delivery. J. Mater. Chem. B 2016, 4, 5803-5808. [CrossRef] 
56. Lin, C.H.; Kumar Kankala, R.; Busa, P.; Lee, C.H. Hydrophobicity-tuned periodic mesoporous organo-silica nanoparticles for photodynamic therapy. Int. J. Mol. Sci. 2020, 21, 2586. [CrossRef]

57. Wu, J.; Bremner, D.H.; Niu, S.; Wu, H.; Wu, J.; Wang, H.; Li, H.; Zhu, L.-M. Functionalized MoS 2 nanosheet-capped periodic mesoporous organosilicas as a multifunctional platform for synergistic targeted chemo-photothermal therapy. Chem. Eng. J. 2018, 342, 90-102. [CrossRef]

58. Qian, X.; Wang, W.; Kong, W.; Chen, Y. Hollow periodic mesoporous organosilicas for highly efficient HIFU-based synergistic therapy. RSC Adv. 2014, 4, 17950. [CrossRef]

59. Doura, T.; Nishio, T.; Tamanoi, F.; Nakamura, M. Relationship between the glutathione-responsive degradability of thiol-organosilica nanoparticles and the chemical structures. J. Mater. Res. 2019, 34, 1266-1278. [CrossRef]

60. Mekaru, H.; Yoshigoe, A.; Nakamura, M.; Doura, T.; Tamanoi, F. Biodegradability of disulfide-organosilica nanoparticles evaluated by soft X-ray photoelectron spectroscopy: Cancer therapy implications. ACS Appl. Nano Mater. 2019, 2, 479-488. [CrossRef]

61. Poscher, V.; Salinas, Y. Trends in degradable mesoporous organosilica-based nanomaterials for controlling drug delivery: A mini review. Materials 2020, 13, 3668. [CrossRef]

(C) 2020 by the authors. Licensee MDPI, Basel, Switzerland. This article is an open access article distributed under the terms and conditions of the Creative Commons Attribution (CC BY) license (http://creativecommons.org/licenses/by/4.0/). 